\title{
Adenocarcinoma mucinoso gástrico em canino
}

\author{
Canine gastric mucinous adenocarcinoma \\ Adenocarcinoma mucinoso gástrico canino
}

\section{Resumo}

Este trabalho descreve os aspectos macroscópicos, histopatológicos e imuno-histoquímicos (IHQ) de um caso de adenocarcinoma mucinoso em um canino da raça Chow-chow, com histórico de vômito e emagrecimento. No exame ultrassonográfico, foi observado espessamento difuso e perda da estratificação da parede do estômago sugestivo de neoplasia gástrica. Após o óbito o animal foi encaminhado ao Laboratório Regional de Diagnóstico da Faculdade de Veterinária da Universidade Federal de Pelotas (LRD/UFPel) para estabelecer o diagnóstico definitivo. Na necropsia havia espessamento difuso da parede do estômago na região antropilórica e porção caudal do corpo do órgão, com achatamento das pregas e ulcerações na mucosa, além de aumento dos linfonodos mesentéricos. Na avaliação histopatológica do estômago foram observadas células epiteliais pleomórficas, com núcleos paracentrais e abundante citoplasma contendo mucina, caracterizando células em anel de sinete. As células neoplásicas comprometiam todas as camadas da parede gástrica, sendo observadas, também, no interior de vasos linfáticos, linfonodos regionais e fígado. $\mathrm{Na}$ coloração de PAS foi evidenciada a presença de mucina no citoplasma das células neoplásicas. Na IHQ houve imunomarcação das células tumorais para citoqueratina. O diagnóstico de adenocarcinoma mucinoso gástrico, neste caso, foi baseado nos achados macroscópicos, histopatológicose confirmado pela IHQ.

Palavras-chave: Carcinoma gástrico; Células em anel de sinete; Imuno-histoquímica; Neoplasias gastrointestinais.

\begin{abstract}
This paper describes the macroscopic, histopathological and immunohistochemical (IHC) aspects of a case of mucinous adenocarcinoma in a Chow-chow canine, with a history of vomiting and weight loss. Ultrasonographic examination revealed diffuse thickening and loss of stratification of the stomach wall suggestive of gastric neoplasia. After death, the animal was sent to the Laboratório Regional de Diagnóstico of the Faculdade de Veterinária of the Universidade Federal de Pelotas (LRD/UFPel) to establish the definitive diagnosis. At necropsy, there was diffuse thickening of the stomach wall in the anthropyloric region and the caudal portion of the body of the organ. , with flattening of the folds and ulcerations in the mucosa, in addition to enlargement of the mesenteric lymph nodes. In the histopathological evaluation of the stomach, pleomorphic epithelial cells were observed, with paracentral nuclei and
\end{abstract}


abundant cytoplasm containing mucin, characterizing signet ring cells. Neoplastic cells affected all layers of the gastric wall, being also observed inside lymphatic vessels, regional lymph nodes and liver. PAS staining showed the presence of mucin in the cytoplasm of neoplastic cells. In IHC there was immunostaining of tumor cells for cytokeratin. The diagnosis of gastric mucinous adenocarcinoma in this case was based on macroscopic findings, histopathological and confirmed by IHC.

Keywords: Gastric carcinoma; Signet ring cells; Immunohistochemistry; Gastrointestinal neoplasms.

\section{Resumen}

Este artículo describe los aspectos macroscópicos, histopatológicos e inmunohistoquímicos (IHC) de un caso de adenocarcinoma mucinoso en un canino de Chow-chow, con antecedentes de vómitos y pérdida de peso. El examen ecográfico reveló engrosamiento difuso y pérdida de estratificación de la pared del estómago sugestivo de neoplasia gástrica. Después de la muerte, el animal fue enviado al Laboratório Regional de Diagnóstico de la Faculdade de Veterinária de la Universidade Federal de Pelotas (LRD/UFPel) para establecer el diagnóstico definitivo. En la necropsia se observó engrosamiento difuso de la pared del estómago en la región antropilórica. y la porción caudal del cuerpo del órgano., con aplanamiento de los pliegues y ulceraciones en la mucosa, además de agrandamiento de los ganglios linfáticos mesentéricos. En la evaluación histopatológica del estómago se observaron células epiteliales pleomórficas, con núcleos paracentrales y abundante citoplasma conteniendo mucina, caracterizando las células en anillo de sello. Las células neoplásicas afectaron todas las capas de la pared gástrica, observándose también en el interior de los vasos linfáticos, los ganglios linfáticos regionales y el hígado. La tinción PAS mostró la presencia de mucina en el citoplasma de las células neoplásicas. En IHC hubo inmunotinción de células tumorales para citoqueratina. El diagnóstico de adenocarcinoma mucinoso gástrico en este caso se basó en hallazgos macroscópicos, histopatológicos y confirmados por IHC.

Palabras clave: Carcinoma gástrico; Células en anillo de sello; Inmunohistoquímica; Neoplasias gastrointestinales.

\section{Introdução}

Adenocarcinomas gástricos são neoplasias malignas de origem epitelial, classificados de acordo com as características histológicas em papilar, tubular, mucinoso e pouco coeso (Galvão et al., 2009; Munday et al., 2017). Nos caninos os adenocarcinomas representam a neoplasia gástrica primária mais comum (Gualtieri et al., 1999; Willard, 2012; Candido et al., 2018), acometendo com maior frequência machos com mais de 10 anos de idade e das raças Rough Collie, Staffordshire Bull Terrier, Chow-chow, Pastor Belga, Lundehund Noruegues, Cairn Terrier e Terrier Branco de West Highland (Sullivan et al., 1987; Bilek et al., 2007; Seim-Wikse et al., 2013; Munday et al., 2017; Koterbay et al., 2020; Hardas et al., 2021).

O adenocarcinoma mucinoso caracteriza-se pela produção excessiva de mucina, sendo um subtipo de ocorrência rara no estômago. Esta variante surge da mucosa, principalmente na lâmina própria e invade as demais camadas do órgão, com frequência fazendo metástase para linfonodos regionais, mesentério, fígado e baço (Swann et al., 2002; Galvão et al., 2009). Os sinais clínicos de tumor gástrico são inespecíficos como inapetência, vômito intermitente ou crônico e perda de peso progressiva com o diagnóstico na maioria dos casos determinado somente em estágio avançado da doença (Sullivan et al., 1987; Von Babo et al., 2012; Seim-Wikse et al., 2013; Abrams et al., 2019), sendo esses sinais clínicos observados mais tardiamente na variante mucinosa (Galvão et al., 2009; Munday et al., 2017). A etiologia dos carcinomas gástricos é desconhecida na maioria dos casos, porém pode estar associada a fatores ambientais e genéticos, assim como em humanos (Scanziani et al., 1991; Lubbes et al., 2009; Munday et al., 2012; Seim-Wikse et al., 2013; Hugen et al., 2016; Munday et al., 2017; Candido et al., 2018). Histologicamente o adenocarcinoma mucinoso é caracterizado por células neoplásicas contendo mucina no citoplasma com núcleo deslocado para a periferia (célula em anel de sinete), além de mucina extracelular (Munday et al., 2017; Hardas et al., 2021).

O objetivo deste trabalho foi descrever os aspectos macroscópicos, histopatológicos e imuno-histoquímicos de um caso de adenocarcinoma mucinoso em um canino da raça Chow-chow. 


\section{Metodologia}

Foi encaminhado ao Laboratório Regional de Diagnóstico, da Faculdade de Veterinária da Universidade Federal de Pelotas (LRD/UFPel) cadáver de um canino, macho, da raça Chow-chow, de 9 anos de idade. O cão apresentava histórico de artrose severa das articulações úmero-radio-ulnar e coxofemoral de ambos os lados e fazia uso contínuo de anti-inflamatório não esteroidal (AINE). O animal morreu 22 dias após apresentar quadro de vômito e emagrecimento, com diagnóstico ultrassonográfico presuntivo de neoplasia gástrica. Durante a necropsia, foram coletados e fixados, em formalina $10 \%$ tamponada, fragmentos de todos os órgãos. Após 48 horas as amostras foram clivadas, processadas, cortadas em secções de $3 \mu \mathrm{m}$ de espessura e coradas pela técnica de hematoxilina e eosina (HE). Cortes selecionados do estômago, linfonodos gástricos e fígado foram submetidos à coloração especial de ácido periódico de Schiff (PAS) e a técnica de imuno-histoquímica (IHQ). Cortes do estômago também foram submetidos a técnica de impregnação pela prata de Warthin-Starry para identificação de Helicobacter spp. Para a IHQ foram utilizados os anticorpos anti-citoqueratina (clone AE1 / AE3) e vimentina (clone V9), ambos na diluição de 1:100. Como cromógeno utilizou-se o 3-3' diaminabenzidine (DAB) e como controle negativo seções do tecido incubadas apenas com o diluente do anticorpo, Phosphate Buffered Saline (PBS). As lâminas foram contra-coradas com hematoxilina de Harris e analisadas em microscopia óptica (Obj. 4x, obj. 10x e obj.40x).

\section{Resultados e Discussão}

No exame de ultrassonografia abdominal total, foi observado espessamento e perda da estratificação da parede gástrica sugestivos de carcinoma gástrico (Figura 1A), corroborando os achados ultrassonográficos descritos na literatura (Kaser-Hotz et al., 1996). Na necropsia observou-se espessamento difuso da parede do estômago na região antropilórica e porção caudal do corpo do órgão, com achatamento das pregas e ulcerações na mucosa (Figura 1B).

Figura 1 - Aspecto ultrassonográfico e macroscópico de adenocarcinoma mucinoso gástrico em canino. 1A - Espessamento e perda da estratificação parietal da parede gástrica (linha central pontilhada), com hipoecogenicidade difusa de parede e irregularidade de superfície mucosa. 1B - Região antropilórica e porção caudal do corpo gástrico apresentando parede espessa, branco-acinzentada e firme (seta), com achatamento das pregas e ulcerações na mucosa (cabeça seta).
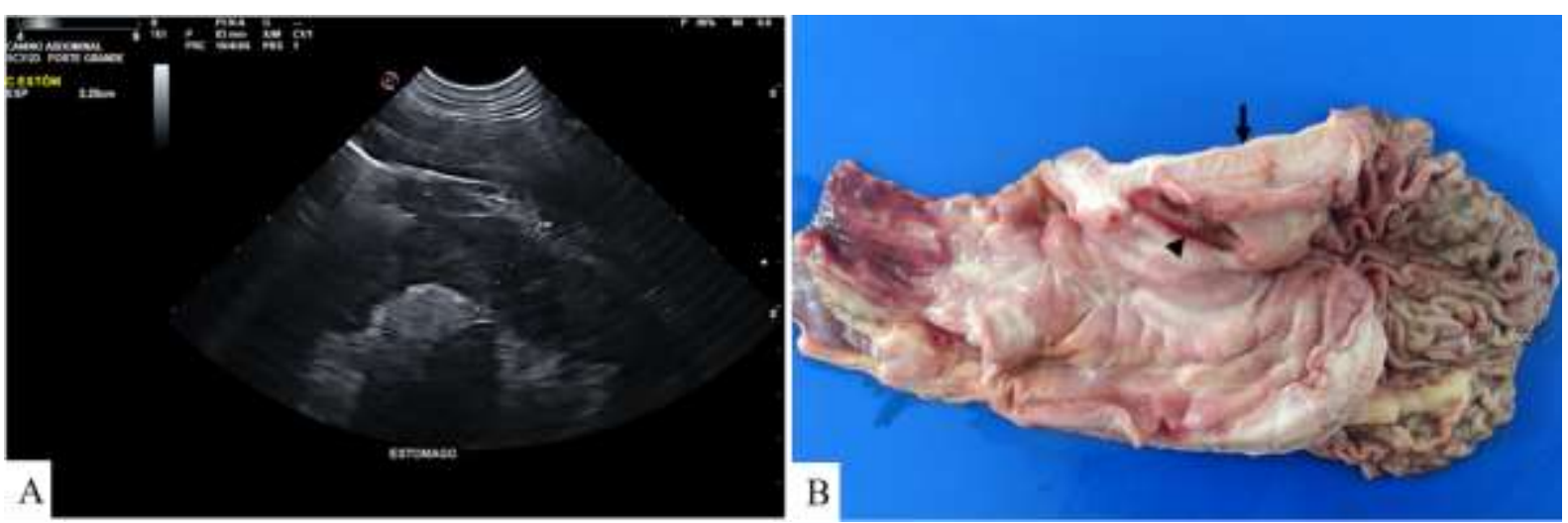

Fonte: Autores.

O intestino delgado apresentava conteúdo sanguinolento em toda sua extensão e havia aumento dos linfonodos mesentéricos.

Em caninos os carcinomas gástricos ocorrem principalmente na curvatura menor e região do piloro (Patnaik et al., 1978; Swann et al., 2002), na maioria dos casos apresentam-se como lesões ulcerativas e/ou massas exofíticas na mucosa (Munday et al., 2017), diferentemente do observado nesse caso que apresentava espessamento difuso da parede gástrica. Esse 
padrão menos frequente ocorre principalmente no subtipo mucinoso (Munday et al., 2017; Hardas et al., 2021). Nessa variante, como no presente caso, a parede apresenta-se firme, branco-acinzentada e pode atingir até $4 \mathrm{~cm}$ de espessura, acometendo inicialmente a região antropilórica (Dell'Orco et al., 2005; Munday et al., 2017).

$\mathrm{Na}$ avaliação histopatopatológica do estômago havia proliferação de células epiteliais pleomórficas, com núcleos paracentrais e abundante citoplasma contendo mucina, caracterizando células em anel de sinete (Figura 2A e 2B). As células neoplásicas comprometiam todas as camadas da parede gástrica, sendo observadas, também, no interior de vasos linfáticos. Nos linfonodos regionais (Figura 2C e 2D) e fígado foram observadas as mesmas células tumorais presentes no estômago.

Figura 2 - Histopatologia de adenocarcinoma mucinoso gástrico em canino. 2A - Corte histológico do estômago evidenciando lagos de mucina e infiltração neoplásica nas camadas mucosa, submucosa e muscular (asterisco) [HE; obj.4x]. 2B - Células em anel de sinete, caracterizadas por núcleo basofílico e paracentral, com citoplasma vacuolizado contendo mucina (seta) na submucosa do estômago [HE; obj.40x]. 2C - Substituição do parênquima normal do linfonodo por células neoplásicas (asterisco) [HE; obj.4x]. 2D - Maior aumento de linfonodo evidenciando células em anel de sinete (seta) em meio a linfócitos [HE; obj.40x].

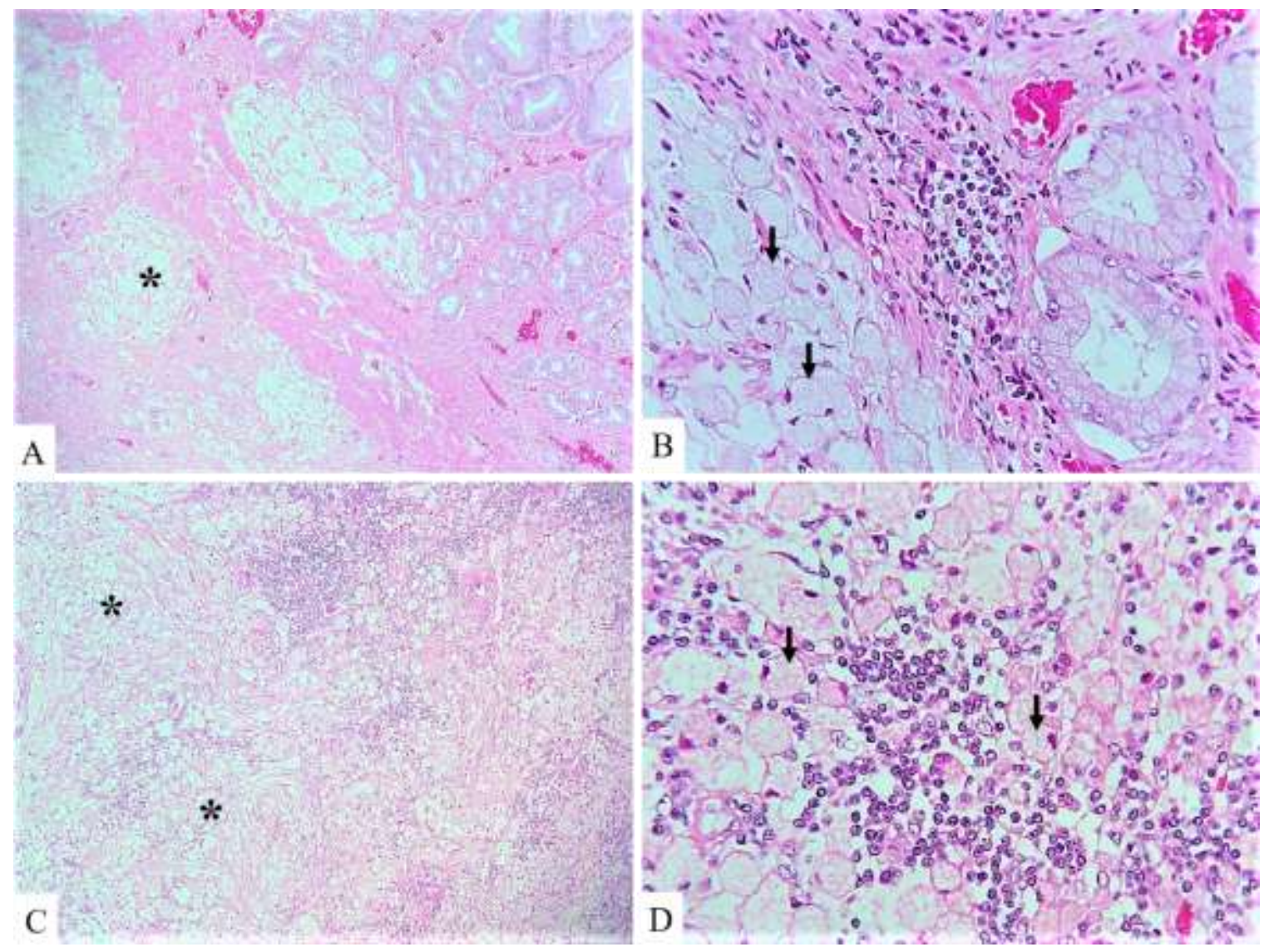

Fonte: Autores.

$\mathrm{Na}$ coloração de PAS foi evidenciada a presença de mucina no citoplasma das células neoplásicas presentes no estômago (Figura 3A e 3B), linfonodos (Figura 3C) e fígado (Figura 3D). 
Research, Society and Development, v. 10, n. 11, e132101119146, 2021

(CC BY 4.0) | ISSN 2525-3409 | DOI: http://dx.doi.org/10.33448/rsd-v10i11.19146

Figura 3 - Coloração de PAS em adenocarcinoma mucinoso gástrico em canino. 3A - Células tumorais fortemente coradas nas camadas submucosa e muscular do estômago (asterisco) [PAS; obj.4x]. 3B - Evidenciação da mucina no interior do citoplasma das células neoplásicas na submucosa gástrica (seta) [PAS; obj.40x]. 3C - Linfonodo com metástase de adenocarcinoma mucinoso (asterisco) [PAS; obj.4x]. 3D - Fígado com células em anel de sinete coradas no PAS (seta) [PAS; obj.40x].
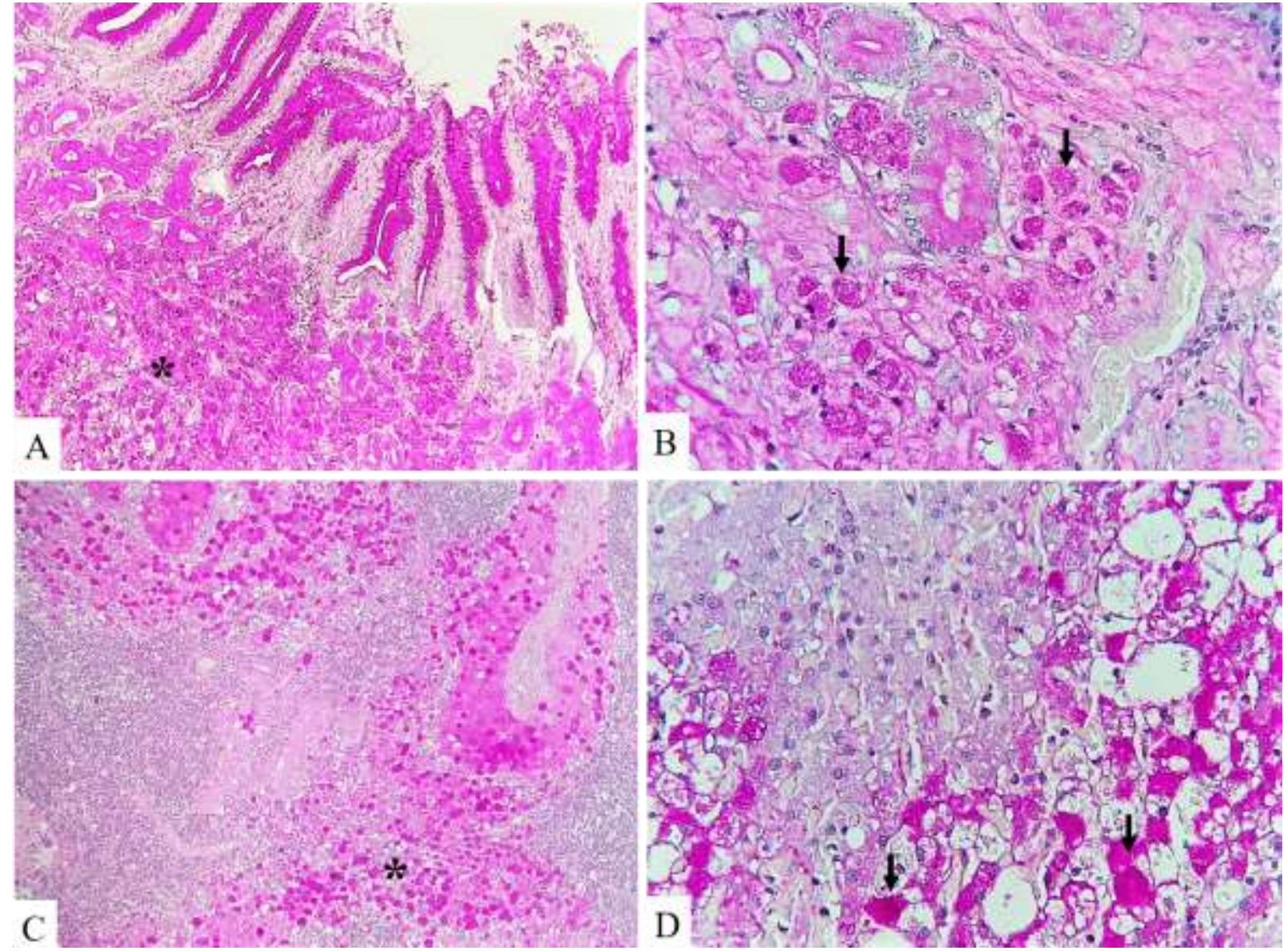

Fonte: Autores.

A técnica de Warthin-Starry não foi significativa para bactérias. Na IHQ houve imunomarcação das células neoplásicas para citoqueratina, confirmando o diagnóstico de adenocarcinoma mucinoso com metástases em linfonodos e fígado (Figura 4A e 4B). Segundo Sullivan et al. (1987) no momento do diagnóstico de carcinoma gástrico em $70 \%$ a $90 \%$ dos casos já ocorreram metástases para linfonodos e outros órgãos. 
Figura 4 - Imuno-histoquímica de adenocarcinoma mucinoso gástrico em canino. 4A e 4B - Imunomarcação positiva das células neoplásicas para citoqueratina (asterisco) [IQH; obj.4x]; (seta) [IHQ; obj.40x].
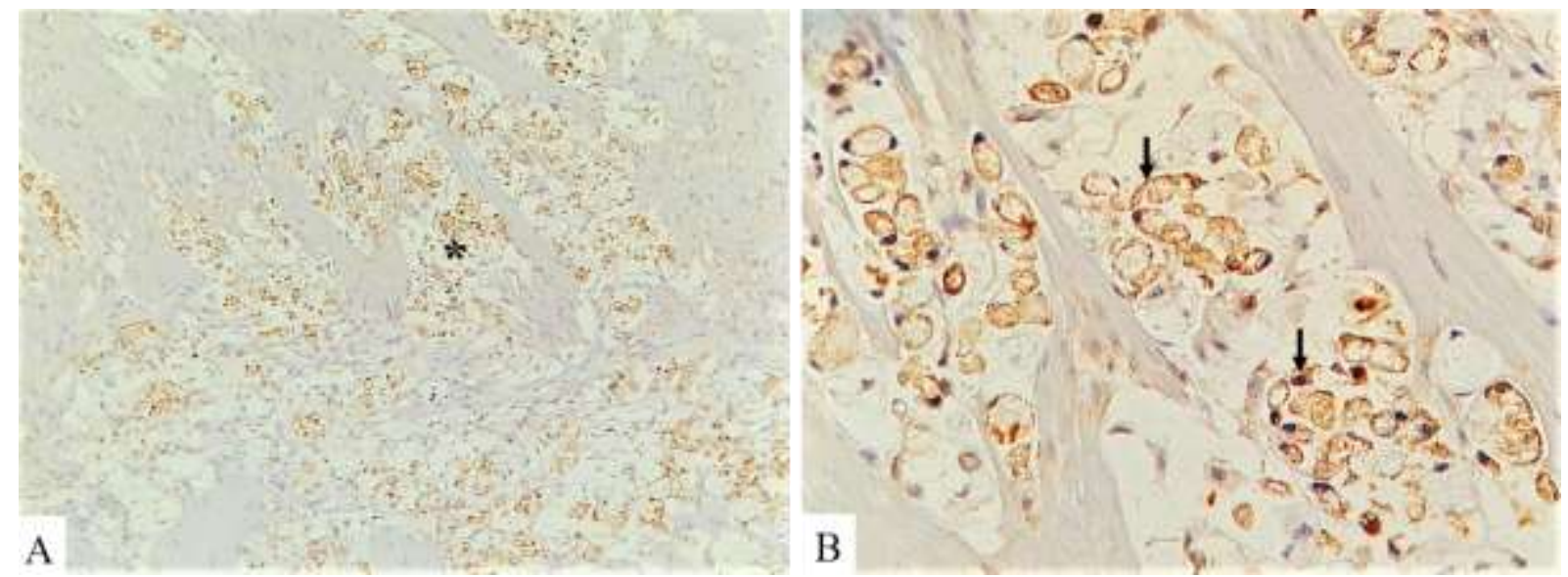

Fonte: Autores.

O diagnóstico de adenocarcinoma mucinoso no presente caso, foi baseado nos achados macroscópicos, histopatológicos e imuno-histoquímicos. No diagnóstico de neoplasias gástricas a IHQ e a coloração especial de PAS são fundamentais para estabelecer a origem das células neoplásicas e determinar o subtipo como mucinoso (Dell'Orco et al., 2005; Munday et al., 2017; Slaviero et al., 2020). A determinação do subtipo histológico de adenocarcinoma apresenta relevância prognóstica (Hsueh et al., 2018). A variante mucinosa é a mais invasiva, com maior potencial metastático e pior prognóstico (Galvão et al., 2009), devido à perda completa da expressão da molécula de adesão E-caderina neste neoplasma (Fearon, 2019; Saito et al., 2020). Na espécie canina a raça Chow-Chow é a mais predisposta a desenvolver a forma mucinosa (Koterbay et al., 2020), como observado no presente caso. A predisposição de raças individuais desenvolverem neoplasias gástricas apoia a teoria dessa ser uma doença hereditária em cães (Hansford et al., 2015; Koterbay et al., 2020).

O quadro clínico de cães com neoplasia gástrica é inespecífico e compatível com doença gastrintestinal na maioria dos casos. A duração dos sinais clínicos pode variar de duas semanas a 18 meses, e a sobrevida após o diagnóstico é de até 35 dias (Swann et al., 2002; Seim-Wikse et al., 2013; Munday et al., 2017). Nos casos de adenocarcinoma mucinoso a manifestação clínica é tardia, sendo estes diagnosticados em estágios mais avançados da doença, com uma sobrevida menor após o diagnóstico (Galvão et al., 2009). No presente caso o canino apresentou sinais clínicos quando mais de 50\% do estômago estava comprometido pelo neoplasma, tendo evolução para morte de apenas 22 dias após o início dos sinais clínicos.

A etiologia do carcinoma gástrico em caninos não foi determinada, porém estudos apontam uma possível influência genética devido a maior susceptibilidade de algumas raças para desenvolver estes neoplasmas (Kelley at al., 2003; Seim-Wikse et al., 2013). Além do fator genético a presença de inflamação crônica também é um fator que influencia diretamente na carcinogenese de tumores gástricos tanto em humanos como em caninos, sendo a infecção por Helicobacter pylori o principal fator de risco para humanos (Morrison, 2012; Balakrishnan et al., 2017). Em caninos a associação de infecção por H. pylori e a ocorrência de neoplasia gástrica não está definida (Koterbay et al., 2020), embora estudo recente tenha demonstrado maior grau de inflamação crônica em neoplasias gástricas quando o H. pylori estava presente (Hardas et al 2021). Entretanto, não foi observado Helicobacter spp. na coloração especial de Warthin-Starry, não sendo esse agente associado a patogênese do neoplasma no presente caso. 


\section{Conclusão}

Tendo em vista a etiologia desconhecida de neoplasias gástricas nesta espécie, é necessário a realização de estudos específicos que possam avaliar a influência de fatores genéticos e de doenças como a infecção por Helicobater spp. na ocorrência dos adenocarcinomas gástricos.

\section{Agradecimentos}

Os autores agradecem a Coordenação de Aperfeiçoamento de Pessoal de Nível Superior (CAPES) (código 001) pelo suporte financeiro e bolsas.

\section{Referências}

Abrams, B., Wavreille, V. A., Husbands, B. D., Matz, B. M., Massari, F., Liptak, J. M., Cray, M. T., Souza, C. H. M., Wustefeld-Janssens, B. G., Oblak, M. L., Su, L., \& Selmic, L. E. (2019). Perioperative complications and outcome after surgery for treatment of gastric carcinoma in dogs: A Veterinary Society of Surgical Oncology retrospective study of 40 cases (2004-2018). Veterinary Surgery, 48:923-932.

Balakrishnan, M., George, R., Sharma, A., \& Graham, D. Y. (2017). Changing trends in stomach cancer throughout the world. Current Gastroenterology Reports, 19(8):36.

Bilek, A., \& Hirt, R. A. (2007). Increased occurrence of race-associated gastric carcinoma in chow-chows. Wiener Tierarztliche Monatsschrift, 94: 71-79.

Candido, M.V., Syrjä, P., Kilpinen, S., \& Spillmann, T. (2018). Canine breeds associated with gastric carcinoma, metaplasia and dysplasia diagnosed by histopathology of endoscopic biopsy samples. Acta Veterinaria Scandinavica, 60:37-46.

Dell’Orco, M., Bertazzolo, W., Vergine, M., Ferla, M., Pozzo, S., Rondena, M., \& Roccabianca, P. (2005). Gastric mucinous adenocarcinoma with cutaneous metastases in a dog: diagnosis by fine-needle aspiration cytology. Journal of Small Animal Practice, 46:449-453.

Fearon, E. R. (2019). Cancer: Context is key for e-cadherin in invasion and metastasis. Current Biology, 29(21):1140-1142.

Galvão, J. F. B., Pressler, B. M., Freeman, L. J., Rohleder, J. J., Burgess, R. C. F., \& Ramos-Vara, J. A. (2009). Mucinous gastric carcinoma with abdominal carcinomatosis and hypergastrinemia in a dog. Journal of the American Animal Hospital Association, 45: 197-202.

Gualtieri, H., Monzeglio, M. G., \& Scanziani E. (1999). Gastric neoplasm. Veterinary Clinics of North America: Small Animal Practice, 29:415-440.

Hardas, A., Suárez-Bonnet, A., Beck, S., Becker, W. E., Ramírez, G. A., \& Priestnall, S. L. (2021). Canine Gastric Carcinomas: A histopathological and immunohistochemical study and similarities with the human counterpart. Animals: An Open Access Journal from, 11(5):1409.

Hansford, S., Kaurah, P., Li-Chang, H., Woo, M., Senz, J., Pinheiro, H., Schrader, K. A., Schaeffer, D. F., Shumansky, K., Zogopoulos, G., Santos, T. A., Claro, I., Carvalho, J., Nielsen, C., Padilla, S., Lum, A., Talhouk, A., Baker-Lange, K., Richardson, S., Lewis, I., Lindor, N. M., Pennell, E., MacMillan, A., Fernandez, B., Keller, G., Lynch, H., Shah, S. P., Guilford, P., Gallinger, S., Corso. G., Roviello, F., Caldas, C., Oliveira, C., Pharoah, P. D., \& Huntsman, D. G. (2015). Hereditary diffuse gastric cancer syndrome: Cdh1 mutations and beyond. JAMA Oncology, 1:23-32.

Hsueh, C. S., Li, W. T., Jeng, C. R., Pang, V. F., \& Chang, H. W. (2018). Diffuse-type gastric mucinous and signet ring cell adenocarcinoma in a captive california king snake (Lampropeltis getula californiae). Journal of Comparative Pathology, 160: 10-14.

Hugen, S., Thomas, R. E., German, A. J., Burgener, I. A., \& Mandigers, P. J. J. (2016). Gastric carcinoma in canines and humans, a review. Veterinary and Comparative Oncology, 15(3):692-705.

Kaser-Hotz, B., Hauser, B., \& Arnold, P: (1996). Ultrasound findings in canine gastric neoplasia in 13 patients. Veterinary Radiology \& Ultrasound, 37:51-56.

Kelley, J. R., Duggan, J. M: (2003). Gastric cancer epidemiology and risk factors. Journal of Clinical Epidemiology, 56:1-9.

Koterbay, A. M., Muthupalani, S., Fox, J. G., \& McNiel, E. A. (2020). Risk and characteristics of gastric carcinoma in the chow chow dog. Canadian Veterinary Journal, 61:396-400.

Lubbes, D., Mandigers, P. J., Heuven, H. C., \& Teske, E. (2009). Incidence of gastric carcinoma in Dutch Tervueren shepherd dogs born between 1991 and 2002. Tijdschrift voor Diergeneeskunde, 134:606-610.

Morrison, W. B. (2012). Inflammation and cancer: a comparative view. Journal of Veterinary Internal Medicine, 26:18-31.

Munday, J. S., Aberdein, D., Cullen, G. D., \& Francês, A. F. (2012). Ménétrier disease and gastric adenocarcinoma in 3 Cairn terrier littermates. Veterinary Pathology, 49(6):1028-1031.

Munday, J. S., Löhr, C. V., \& Kiupel, M. (2017). Tumors of the Alimentary Tract. In: Meuten D. J. (Ed). Tumors in Domestic Animals. 5th. edn. Ames: Iowa State University Press, pp.499-631.

Patnaik, A. K., Hurvitz, A. I., \& Johnson, G. F. (1978). Canine gastric adenocarcinoma. Veterinary Pathology, 15:600-607. 
Research, Society and Development, v. 10, n. 11, e132101119146, 2021

(CC BY 4.0) | ISSN 2525-3409 | DOI: http://dx.doi.org/10.33448/rsd-v10i11.19146

Saito, T., Chambers, J. K., Nakashima, K., Nibe, K., Ohno, K., Tsujimoto, H., Uchida, K., \& Nakayama, H. (2020). Immunohistochemical analysis of betacatenin, E-cadherin and p53 in canine gastrointestinal epithelial tumors. Journal of Veterinary Medical Science, 82:1277-1286.

Scanziani, E., Giusti, A. M., Gualtieri, M., \& Fonda, D. (1991). Gastric carcinoma in the Belgian shepherd dog. Journal of Small Animal Practice, 32:465-469.

Seim-Wikse, T., Jörundsson, E., Nodtvedt, A., Grotmol, T., Bjornvad, C. R., Kristensen, T. A., \& Skancke, E. (2013). Breed predisposition to canine gastric carcinoma — A study based on the Norwegian canine. Acta Veterinaria Scandinavica, 55:25.

Slaviero, M., Argenta, F. F., Ehlers, L. P., De Lorenzo, C., Pavarini, S. P., Driemeier, D., \& Sonne, L. (2020). Primary nonlymphoid gastrointestinal neoplasms in dogs in Rio Grande do Sul. Pesquisa Veterinária Brasileira, 40(1):61-71.

Sullivan, M., Lee, R., Fisher, E. W., Nash, A. S., \& Mccandlish, I. A. P. (1987). A study of 31 cases of gastric carcinoma in dogs. Veterinary Record, 120: 7983.

Swann, H. M., \& Holt, D. E. (2002). Canine gastric adenocarcinoma and leiomyosarcoma: a retrospective study of 21 cases (1986-1999) and literature review. Journal of the American Animal Hospital Association, 38: 157-164.

Von Babo, V., Eberle, N., Mischke, R., Meyer-Lindenberg, A., Hewicker-Trautwein, M., Nolte, I., \& Betz, D. (2012). Canine non-hematopoietic gastric neoplasia. Epidemiologic and diagnostic characteristics in $38 \mathrm{dogs}$ with post-surgical outcome of five cases. Tierarztl Prax Ausg K Kleintiere Heimtiere, 40(4):243-249.

Willard, M. D. (2012). Alimentary neoplasia in geriatric dogs and cats. Veterinary Clinics of North America: Small Animal Practice, 42:693-706. 